\title{
Using Artificial Neural Networks for Recognition of Control Chart Pattern
}

\author{
El Farissi. O, \\ Laboratory LMIT, FSA, \\ BP8106 Cite Dakhla \\ Agadir, Morocco
}

\author{
Moudden. A, \\ Laboratory LMIT, FSA, \\ BP8106 Cite Dakhla \\ Agadir, Morocco
}

\author{
Benkachcha. S, \\ Laboratory LISER, ENSEM, \\ Km 7 BP 8118 Route El Jadida \\ Casablanca, Morocco
}

\begin{abstract}
Control charting is an important tool in SPC to improve the quality of products.

Unnatural patterns in control charts assume that an assignable cause affecting the process is present and some actions must be applied to overcome the problem. By its automatic and fast recognition ability the neural network provide best performance to immediately recognize process trends. In this paper, a neural network model is used to control chart pattern recognition (CCPR).
\end{abstract}

Several forms of architectures have been tested and the results point out a network configuration which leads to excellent quality of recognition.

\section{General Terms}

\begin{tabular}{|c|c|c|}
\hline SPC & $=$ & Statistical Process Control; \\
\hline CCPR & $=$ & Control charts pattern recognition; \\
\hline $\mathrm{CCP}$ & $=$ & Control charts pattern; \\
\hline $\mathrm{CC}$ & $=$ & Control charts; \\
\hline NOR & $=$ & Normal; \\
\hline IT & $=$ & Increasing trend; \\
\hline US & $=$ & Upward shift; \\
\hline $\mathrm{ANN}(\mathrm{s})$ & $=$ & Artificial Neural Network(s); \\
\hline MLP & $=$ & Multilayer Perceptron; \\
\hline $\begin{array}{l}\mathrm{LM} \\
\text { algorithm }\end{array}$ & $=$ & Levenberg -Marquardt back propagation \\
\hline $\begin{array}{l}\text { GDA } \\
\text { rate back }\end{array}$ & $\begin{array}{l}= \\
\text { prc }\end{array}$ & $\begin{array}{l}\text { Gradient descent with adaptive learning } \\
\text { on; }\end{array}$ \\
\hline SSE & $=$ & Error Sum of Squares; \\
\hline MSE & $=$ & Mean Square Error; \\
\hline
\end{tabular}

\section{Keywords}

Artificial Neural Networks (ANN), Statistical Process Control (SPC), Control Charts (CC), Control Charts Pattern (CCP).

\section{INTRODUCTION}

Statistical Process Control (SPC) has been widely used for monitoring the production process. Control chart pattern recognition is the most commonly SPC tools used for problem identification in processes due to special causes. Indeed, traditional Control charts use only the control limits to detect changes in the process according to the latest data sets. But the nature of the evolution of these data is not taking into account.

Otherwise the improvement of the detection quality by implementing control rules is limited by false alarms that arise by the simultaneous application of these rules.

There are three main types of patterns that commonly appear in CCPs: normal (NOR), increasing trend (IT), and upward shift (US), as is shown in Figure 1.

The NOR pattern indicates that the process is operating under control. All other types of patterns are unnatural and assume that an assignable cause affecting the process is present.

For the high speed production, the Advances in measurement technology provide a wide possibility to record and plot realtime data. In the last decade, neural network has emerged as a practical approach for automatically recognizing control chart patterns (CCPs). In this field, a large amount of research [1][2]-[3]-[4] has been carried out using of feedforward network architectures, among which multilayered perceptrons (MLPs) are the most commonly adopted.

This paper presents a contribution in CCPs by using an MLP in order to improve the ability of pattern detection. The MLP is trained for different structures, and several learning rules used to adjust the ANN weights have been evaluated. The best configuration and the most accurate algorithm are retained.

The rest of the paper is organized as follow: The second section will review the literature in CCP and the use of Artificial Neural Networks in this area. The third section will present the neural network design for pattern recognition. In the fourth section, the results obtained will be discussed. The last section will review the main results of the paper.

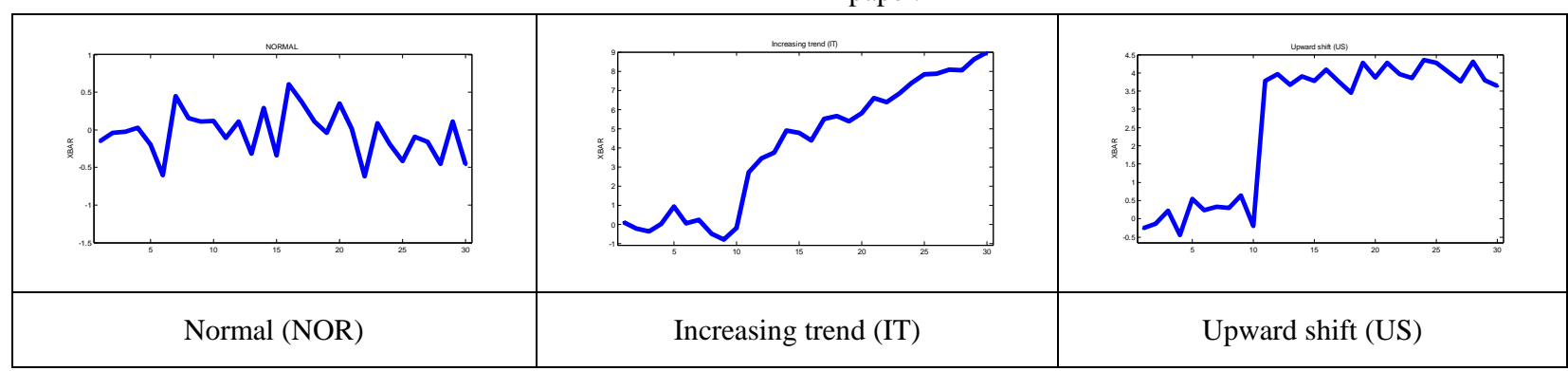

Fig 1.Three types of basic CCPs 


\section{NEURAL NETWORKS FOR CONTROL CHARTS PATTERN RECOGNITION}

Anagun [5] used a backpropagation network (BPN) to recognize patterns in SPC. The training data were organized in two different ways: direct representation and histogram representation. The results show that the later method provided higher performance than the direct representation.

Moreover, Guh and Tannock [6] dealing with single patterns such as sudden shifts, linear trends or cyclic patterns, investigated the use of an (BPN) to recognize concurrent CCPs where several pattern exist together.

N.V.N. Kiran et al. [7] evaluated the relative performance of the five training algorithms. The structures for CCP recognizer tested in this study comprise an input layer, one hidden layer and an output layer. The best result is trained with a traindx algorithm.

Feedforwardneural networks are organized in layers and this architecture is often known as MLP (multilayer perceptron) [8]. An example of feedforward neural network consisting of an input layer, a hidden layer, and an output layer is shown in figure 2 .

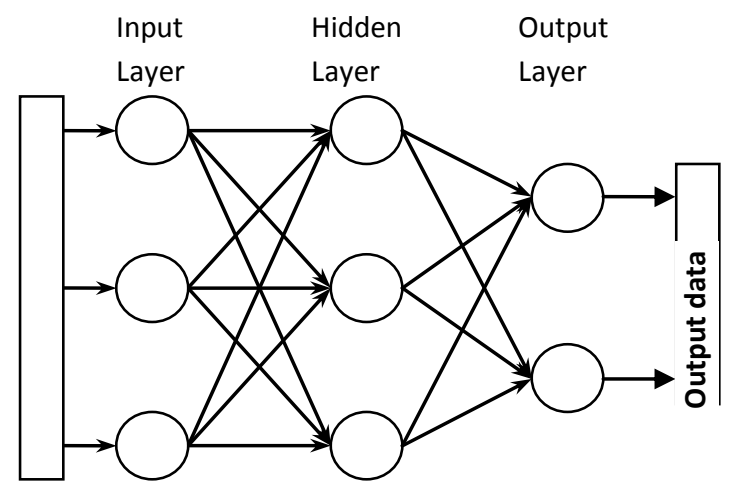

Fig 2. Basic structure of multilayer perceptron

Each node in a neural network is a processing unit which contains a weight $\left(w_{i j}\right)$ and summing function followed by a transfert function $(f)$. Fig3.

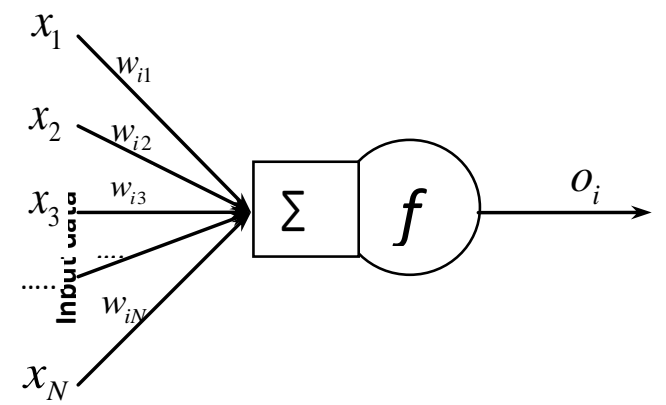

Fig 3. Single neuron with $\mathbf{N}$ inputs

The summer output, referred to as the total synaptic input of the neuron, is given by the inner product of the input and weight vectors : $\sum w_{i j} x_{j}$

The output value of the neuron is given by:

$$
o_{i}=f\left(\sum w_{i j} x_{j}\right)
$$

Where:

- $f($.$) is the transfer function,$

- $w_{i j}$ is the connection weight between node $j$ and node $i$,

- $x_{j}$ the input signal from the node $j$.

- $o_{i}$ is the output of the neuron $i$,

The general process responsible for training the network is mainly composed of three steps: feed forward the input signal, back propagate the error and adjust the weights.

The back-propagation algorithm tray to improve the performance of the neural network by reducing the total error which can be calculated by:

$$
S E=\frac{1}{2} \sum_{p} \sum_{j}\left[o_{j p}-d_{j p}\right]^{2}
$$

Where:

- $\quad S E$ is the square error,

- $\quad p$ is the number of applied patterns,

- $d_{j p}$ is the desired output for $j^{\text {th }}$ neuron when $p^{\text {th }}$ pattern is applied and

- $\quad o_{j p}$ is the $j^{\text {th }}$ neuron output.

\section{NEURAL NETWORK DESIGN FOR PATTERN RECOGNITION}

\subsection{Sample patterns}

Sample patterns to be classified are usually groups of measurements or observations coming from a real manufacturing process. Since real process containing all type of patterns is not available, simulated data are often used [7][8].The following equations, shown in Table (1), were used to generate the data points for the various patterns.

Table 1. Equations for generating various patterns

\begin{tabular}{|c|l|}
\hline Patterns & \multicolumn{1}{|c|}{ Equations } \\
\hline Normal & $\mu+\sigma * \operatorname{randn}([p, n])$ \\
\hline Trend increasin & $\mu+\sigma * \operatorname{randn}([p, n])+I * g$ \\
\hline Shift up & $\mu+\sigma * \operatorname{randn}([\mathrm{p}, \mathrm{n}])+\mathrm{K} * \mathrm{~s}$ \\
\hline
\end{tabular}

where :

- $\mu$ :is the nominal mean value of the process variable;

- $\sigma$ :is the standard deviation of the process variable;

- $g$ :is the gradient of an increasing trend pattern or a decreasing trend pattern;

- $\operatorname{randn}(p, n)$ is a Matlab Function that generates an p-by-n matrix of random normally distributed with mean $\mu=0$,variance $\sigma^{2}=1$, and standard deviation $\sigma=1$ ( $\mathrm{n}$ is the size of the observation window and $\mathrm{p}$ is the number of observations);

- $s$ :indicates the shift position in an upward shift pattern and a downward shift pattern( $\mathrm{s}=0$ before the shift and 
$\mathrm{s}=1$ at the shift and thereafter)

- $\mathrm{a}$ : is the amplitude of cyclic variations in a cyclic pattern;

- $T$ :is the period of a cycle in a cyclic pattern .

In trend and shift patterns data generation the first $\mathrm{p} / 3$ samples have a normal distribution $\left(\mathrm{K}_{\mathrm{ij}}\right.$ and $\mathrm{I}_{\mathrm{ij}}$ are set to $0)$, then after $K_{i j}=1$ and $I_{i j}=1$ :

$$
\begin{aligned}
& \underset{p \times n}{[K]:} \quad K_{i j}=0 \quad \text { for } i \leq p / 3, \text { and } K_{i j}=1 \quad \text { for } i>p / 3 \\
& \underset{p \times n}{[I]:} \quad I_{i j}=0 \quad \text { for } i \leq p / 3, \text { and } I_{i j}=i \quad \text { for } i>p / 3 .
\end{aligned}
$$

For each type of the six $\mathrm{CCPs},(\mathrm{n} * \mathrm{p})$ samples data were generated using the following values of parameters shown in table 2 .

Table 2. Values of Parameters Adopted To Generate Patterns

\begin{tabular}{|c|c|}
\hline Patterns & Parameter's values \\
\hline Normal & $\mu=0, \sigma=1$ \\
\hline Increasing Trend & $\mathrm{g}=0.3$ \\
\hline Shift Up & $\mathrm{s}=4$ \\
\hline
\end{tabular}

\subsection{Neural Network Design}

In this study the MLP used consist of an input layer, an output layer and two hidden layers as shown in figure 4. In input layer, the $\mathrm{n}$ first nodes correspond to the sample size used for process control. The remaining three nodes represent the statistics of the $\mathrm{n}$ observations which are their average $\bar{X}$, their range $R$ and their standard deviation $S$ (a total of $n+3$ inputs) [9].A single neuron was required for output layer with the normalized coding shown in table 3 .

Table 3.Values of Output Targets Related To Each Pattern

\begin{tabular}{|c|c|}
\hline Patterns & coded output $=$ Target \\
\hline Normal & 0.5 \\
\hline Increasing Trend & 0.1 \\
\hline Shift Up & 0.9 \\
\hline
\end{tabular}

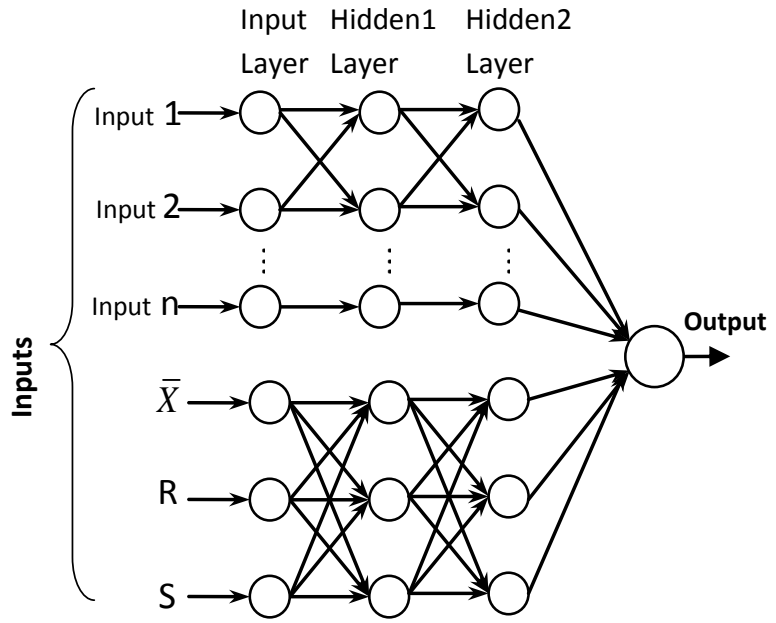

Fig 4.The structure of multilayer perceptron used for CCPs
The training input is an $(\mathrm{n}+3)$-by-(6xp)matrix illustrated as follow (Fig. 5).

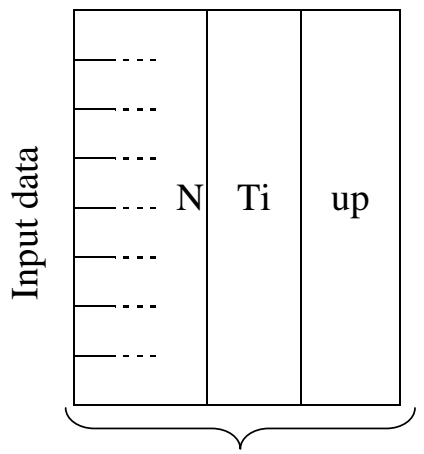

$(3 \times p)$

Fig 5. Training input data structure design

\section{TESTS AND RESULTS}

Neural Network Matlab's Toolbox provides a complete environment to design, train, visualize, and simulate neural networks. The number of neurons in the hidden layers, the activation function in each layer, and the training algorithm are a set of parameters needed to complete the definition of the MLP designed.The most common approach to select the optimal number of hidden nodes and other neural network parameters is via experiment or by trial-and-error [10].

This section presents results and comparisons of the performance between neural networks recognizers trained and tested using three algorithms for several sizes in hidden layers.

To determine the optimal MLP topology and training algorithm, the coefficient of correlation (R)between actual targets and predicted targets and the mean sum of squares of the network errors (MSE) is used [11].

$$
M S E=S S E /(n-p)(6)
$$

Where:

- $\quad$ SSE is the summed square of residuals

- $\quad n$ is the number of observations

- $\quad p$ is the number of terms currently included in the model.

At the start, we can confirm that the choice of tansig as activation function for all layers and an MLP architecture with two hidden layers yields interesting results. To increase accuracy of the MLP, we tried three training algorithms implemented for several numbers of nods in hidden layers. The training algorithms tested here are:

- The back propagation (GD) algorithm: It is a gradient steepest descent method, implemented with traingd function in toolbox of MATLAB.

- Gradient descent with adaptive learning rate back propagation (GDA) algorithm: traingda function in MATLAB toolbox.

- Levenberg-Marquardt back propagation(LM) algorithm: trainlm function in MATLAB toolbox.

Table 4 below present the comparison of MSE performance between actual targets and predicted targets. For each training algorithm, the value of MSE performance is calculated by changing the number of neuron in the hidden layers. 
Table 4.Comparison Of The MSE Performance

\begin{tabular}{|c|c|c|c|}
\hline NNHL & Traingd & traingda & trainlm \\
\hline $7 \times 3$ & 0.0405 & 0.0159 & 0.0256 \\
\hline $8 \times 3$ & 0.0292 & 0.0236 & $1.68 \mathrm{e}-11$ \\
\hline $9 \times 3$ & 0.0335 & 0.0215 & $4.91 \mathrm{e}-7$ \\
\hline $10 \times 3$ & 0.0277 & 0.0230 & 0.0239 \\
\hline $11 \times 3$ & 0.0357 & 0.00961 & 0.0246 \\
\hline $12 \times 3$ & 0.0327 & 0.0251 & $1.46 \mathrm{e}-5$ \\
\hline $13 \times 3$ & 0.0304 & 0.0211 & 0.025 \\
\hline
\end{tabular}

Table 4 show the lowest MSE are obtained for simple size $\mathrm{n}=8$ and for LM algorithm (trainlm function in MATLAB toolbox).

This sample size and LM algorithm are then selected for the execution of Neural Network.

In figure 6, the predicted target for NNt Recognizer selected is practically identical with the actual target.

The execution shows that an MLP with 13 neurons in the first hidden layer and 3 neurons in the second hidden layer trained with LM back propagation algorithm gives better performance in patterns recognition problems.

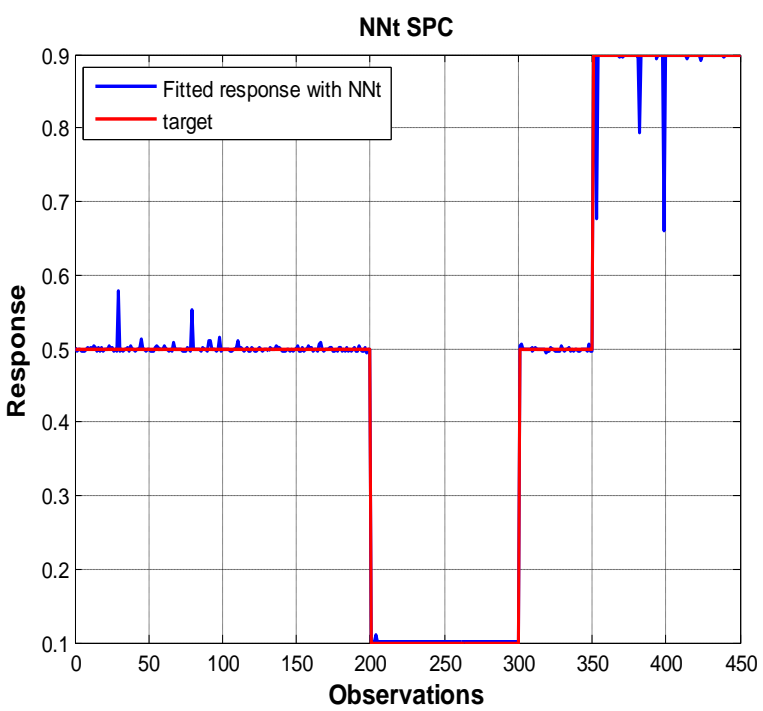

Fig 6. Comparison between actual targets and predicted targets for 13-13-3-1 NNt recognizer trained with $\mathrm{LM}$ algorithm

\section{CONCLUSION}

In this paper, the objective was to improve the quality of the $\mathrm{NNt}$ in CCP recognition. To evaluate its relative performance, the MLP was trained for different structures and training algorithms. The results show that an MLP with 13 neurons in the first hidden layer and 3 neurons in the second hidden layer trained with LM back propagation algorithm provided the best performance in patterns recognition problems.
The work will be extended to study other patterns namely: Decreasing trend (DT), Downward shift (DS) and cyclic (CYC) taking into account other criteria such as the correlation coefficient and the number of iterations.

\section{REFERENCES}

[1] N.V.N. Indra Kiran, Effective Control Chart Pattern Recognition Using Artificial Neural Networks, IJCSNS International Journal of Computer Science and Network Security, VOL.10 No.3, March 2010.

[2] W. Laosiritaworn and T.t Bunjongjit,Classification Techniques for Control Chart Pattern Recognition: A Case of Metal Frame for ActuatorProduction, Chiang Mai J. Sci, 40(4) : 701-712, 2013.

[3] A. D. karaoglan,An Integrated Neural Network Structure for RecognizingAutocorrelated and Trending Processes, Mathematical and Computational Applications, Vol. 16, No. 2, pp. 514-523, 2011.

[4] M. A. Hadiyat and K. R. Prilianti, Comparing Statistical Feature and Artificial Neural Networks forControl Chart Pattern Recognition: A Case Study,The 3rd International Conference on Technology and Operations Management "Sustaining Competitiveness through Green Technology Management"Bandung - Indonesia, July 46, 2012

[5] A. S. Anagun, A Neural Network Applied to Pattern Recognition in Statistical Process Control, Computers \& Industrial Engineering 35, 185-188, 1998.

[6] R. S. Guh and J. D. T. Tannock, Recognition of Control Chart Concurrent Patterns Using a Neural Network Approach, International Journal of Production Research 37, 1743-1765, 1999.

[7] N.V.N. IndraKiran,M.Pramiladevi and G.Vijaya Lakshmi, Training Multilayered Perceptrons for Pattern Recognition: A Comparative Study of Five Training Algorithms, IMECS, Vol. I, March 2011.

[8] B. M.Wilamowski, Neural Network Architectures. Industrial Electronics Handbook (vol. 5 - Intelligent Systems, 2nd Edition, chapter 6, pp. 6-1 to 6-17, CRC Press, 2011).

[9] A. E .Smith, X-bar and R control chart interpretation using neural computing, International Journal of Production Research, 32(2), 309-320, 1994.

[10] G. Zhang, B. E. Patuwo, and M.Y. Hu, Forecasting With Artificial Neural Networks, The state of the art, International Journal of Forecasting.Vol.14, , p 35-62, 1998.

[11] S. Makridakis, and M. Hibon, Evaluating Accuracy (or Error) Measures, Working Paper, INSEAD Fontainebleau, France, 1995

\section{AUTHORS' PROFILE}

Omar El farissi was born in 1972 in Morocco.Hereceived his MASTER in Laboratory of Mechanics, precedesof energy and environments(LMP2E), of ENSA-Agadir in 2012. In $2012 \mathrm{He}$ joined the Laboratory ofMetrology and information's treatment (LMIT) of the FSAIbn Zohr University, Agadir, Morocco. His current research field is SPC and her industrial applications.

Ali Moudden was born in 1958 in Morocco. He obtained (1987) the " doctorat d'état" in physics at the University of 
Caen (France). He founded with other colleagues in 1991, the laboratory of instrumentation and measurements at the Faculty of Sciences of Agadir (Morocco), that became in 2005 the laboratory of metrology and information processing. Laboratory activities are focused mainly on the development of purely physical techniques for evaluation of materials and monitoring of processes. He was director of higher school of technology of Agadir during the period 2005 - 2011.
Said Benkachcha was born in 1972 in Morocco.Hereceived his DESA in Laboratory of Mechanics of Structures and Materials, LMSM, of ENSEM - Casablanca in 2006. In 2011 $\mathrm{He}$ joined the Laboratory of Computer Systems and Renewable Energy (LISER) of the ENSEM Hassan II University, Casablanca, Morocco. His current research field is demand forecasting and collaborative warehouse management. 